\title{
Condition Monitoring of Forward Curved Centrifugal Blower Using Coast Down Time Analysis
}

\author{
G. R. Rameshkumar, ${ }^{1}$ B. V. A. Rao, ${ }^{2}$ and K. P. Ramachandran ${ }^{3}$ \\ ${ }^{1}$ Department of Mechanical \& Industrial Engineering, Caledonian College of Engineering, P.O. Box 2322, CPO 111 Seeb, Oman \\ ${ }^{2}$ Advisor to Chancellor, VIT University, Vellore, Tamil Nadu 632 014, India \\ ${ }^{3}$ Associate Dean (PGS and R), Caledonian College of Engineering, P.O. Box 2322, CPO 111 Seeb, Oman
}

Correspondence should be addressed to G. R. Rameshkumar, ramesh@caledonian.edu.om

Received 19 April 2010; Accepted 17 August 2010

Academic Editor: R. Amano

Copyright (c) 2010 G. R. Rameshkumar et al. This is an open access article distributed under the Creative Commons Attribution License, which permits unrestricted use, distribution, and reproduction in any medium, provided the original work is properly cited.

Mechanical malfunctions such as, rotor unbalance and shaft misalignment are the most common causes of vibration in rotating machineries. Vibration is the most widely used parameter to monitor and asses the machine health condition. In this work, the Coast Down Time (CDT), which is an indicator of faults, is used to assess the condition of the rotating machine as a condition monitoring parameter. CDT is the total time taken by the system to dissipate the momentum acquired during sustained operation. Extensive experiments were conducted on Forward Curved Centrifugal Blower Test Rig at selected cutoff speeds for several combinations of combined horizontal and vertical parallel misalignment, combined parallel and angular misalignment, as well as for various unbalance conditions. As mechanical faults increase, a drastic decrease in CDT is found and this is represented as CDT reduction percentage. A specific correlation between the CDT reduction percentage, level of mechanical faults, and rotational cutoff speeds is observed. The results are analyzed and compared with vibration analysis for potential use of CDT as one of the condition monitoring parameter.

\section{Introduction}

Condition monitoring of rotating machinery helps in detection and prediction of faults at the early stages, which proves to be beneficial in terms of increased availability of machine, reduced down time, and avoidance of accidents. Vibration is one of the widely used monitoring parameter to assess the health condition of the rotating machine. Periodic or continuous monitoring of vibration reveals the actual condition of the machine [1]. Mechanical malfunctions such as, rotor unbalance and shaft misalignment are the most common causes of vibration in rotating machineries. Unbalance is a condition where the center of mass (rotor disc, blower impeller) is not coincident with the center of rotation (shaft). Excessive unbalance can lead to fatigue of machine components and can cause wear in bearings or internal rubs that can damage seals and degrade machine performance. The unbalance part of the rotor rotates at the same speed as the rotor and therefore the force caused by the unbalance is synchronous [2]. Shaft misalignment occurs when the centerlines of rotation of two or more machinery shafts are not in line with each other. There are two types of misalignments: parallel misalignment occurs when the shaft centerlines of the two machines are parallel, but offset to each other, and angular misalignment occurs when the shaft centerlines are not parallel, but inclined to each other. Misalignment in shafts produces high radial loads in one or more bearings [3]. In actual operating environments in industries, achieving perfect balance and perfect alignment between driving and driven shafts is very difficult and a minute amount of unbalance and misalignment conditions are always present. Vibration signatures are widely used as a useful tool for studying progressive machine mechanical malfunctions, and also form the baseline signature for further comparative monitoring to detect mechanical faults [4]. 
When the power supply to any rotating system is cutoff, the system begins to lose the momentum gained during sustained operation and finally comes to rest. The behavior of the system during this period is known as the Coast Down Phenomenon (CDP). The exact time period between the power cutoff time and the time at which the rotor stops is called Coast Down Time $[5,6]$. The CDT is the total time taken by the system to dissipate the momentum acquired during sustained operation. An extensive investigation conducted on vertical rotors supported by rolling element bearings established that CDT monitoring could be used as a health monitoring, quality control, and maintenance tool [7]. The CDP is inherent of a system and the CDT depends on many factors like inertia forces of the system components and tribological behavior of rotating system components such as, bearings, seals, carbon brushes; it also depends on operating conditions and environmental effects such as fluid drag. Some researchers $[8,9]$ have reported experiments conducted on rotor system to evaluate the bearing lubrication for different operating conditions and the influence of the rotor unbalance response on CDT. It was found that CDT could be used as an effective diagnostic parameter and could provide pertinent information regarding the tribological behavior, degradation, and the effectiveness of lubrication. Prabhu [10] and Arumugam et al. [11] evaluated the performance of the misaligned cylindrical and three-lobe journal bearings, and found that CDT decreases with the increase of misalignment. This is because both the bearing friction and the power loss increase with the increase in misalignment. Increase in misalignment will cause an increase in coefficient of friction and reduces the film thickness resulting in an increase in damping factor. Sekhar and Prabhu [12] established in their work that there will be severe vibrations in rotating machines with improper alignment of shafts and proposed a theoretical model of a rotor-bearing system using higher-order Finite Element Method (FEM) analysis. The experimental investigation conducted on a flexible rotor hydrodynamic journal bearing has revealed the influence of misalignment on coast down time [13]. Ramachandran et al. [14] conducted experiments to approach the misalignment using vibration, orbit, and CDT phenomenon and they found that improper alignment leads to extensive vibration and noise and the increased misalignment reduces the CDT. Prabhakar et al. [15] analyzed the transient response of a misaligned rotorcoupling-bearing system passing through the critical speed using the finite element method for flexural vibrations and found that the subcritical speeds at one-half, one-third, and one-fourth of the critical speed. The mathematical model constructed for the effect of misalignment on magnetic coupling [16] derived a set of nonlinear equations for parallel misalignment effect [17] between two rotating shafts and two rotor segments with angular misalignment [18]. Extensive experimental studies were made by researchers [19-23] on unbalance and misalignment $[24,25]$, misaligned rotorball bearing systems $[26,27]$, rigidly coupled rotors $[28$, 29], and pointing complexity of diagnosis for misalignment and unbalance using vibration analysis. In particular, the literature on the CDT analysis for considering the effect of misalignment and unbalance in rotating machinery is hardly found and these are to be given due consideration [30]. CDT analysis is a powerful parameter for studying the significant machine health particularly when the rotor systems are supported with bearings. It can be used as a consistent guide to assess the condition of the system. However, it has not received much attention by researchers. CDT, together with the vibration analysis, could be used as a powerful diagnostic tool for condition monitoring of rotating machineries.

Extensive experimental investigations were carried out by the investigators referred to earlier on De Laval (Jeffcott) rotor system supported between two bearings. However, an industrial environment to assess the CDT as a condition monitoring parameter was not explored. Forward curved blowers also termed as Squirrel Cage centrifugal blowers produce very low noise level and are widely used for industrial application due to their compactness [31]. In this paper, an attempt is made to investigate the use of CDT analysis as one of the condition monitoring parameter in a Forward Curved (FC) centrifugal blower to assess the effect of misalignment and unbalance for understanding the mechanical behavior of system under simulated industrial environment. Further, $\mathrm{CDT}$ analysis is compared with vibration signature analysis for identifying the level of misalignment and the severity of unbalance. Vibration spectrums for both misalignment and unbalance conditions are presented and discussed.

\section{Experimental Test Rig and Instrumentation}

The schematic diagram of Forward Curved Centrifugal Blower experimental test rig [32] used for this investigation is shown in Figure 1. A Forward Curved (FC) centrifugal blower is mounted on a shaft with length $315 \mathrm{~mm}$ and diameter of $20 \mathrm{~mm}$ at the center position of $190 \mathrm{~mm}$ between two anti-riction bearings. The specifications of the blower used for this investigation are given in Table 1. The shaft is supported between two Z-type SKF antifriction ball bearings. The blower shaft is connected through an electromagnetic coupling to a variable speed DC motor (speed $3000 \pm 5 \% \mathrm{rpm}$ ). One side of motor shaft is supported by one each of Z-type and P Block (self-aligning) bearings and other side of motor shaft is supported by $\mathrm{P}$ Block bearing. Blower setup and two Z-type bearings housing frames are bolted to an adjustable steel plate of size $400 \mathrm{~mm}$ $\times 300 \mathrm{~mm} \times 15 \mathrm{~mm}$ to introduce the required misalignment condition between the blower shaft and motor shaft. This assembly is mounted on a main heavy steel frame with four adjustable screws at four corner ends of the steel plate and a lead screw at the bottom, for horizontal movement of the steel plate. The contact surface of steel plate assembly and main steel frame are made perfectly flat and smooth for accurate alignment between the two contact surfaces. The whole test rig unit is mounted on a heavy steel framework, which is clamped to a concrete foundation with antivibration rubber pads. Two inductive proximity sensors are used to measure the speeds of blower and motor independently. Load cell sensor of CZL-601 type, with a capacity of $30 \mathrm{~kg}$, is mounted at the motor end to measure torque in $\mathrm{Nm}$. A speed controller knob is used to vary the voltage to adjust 

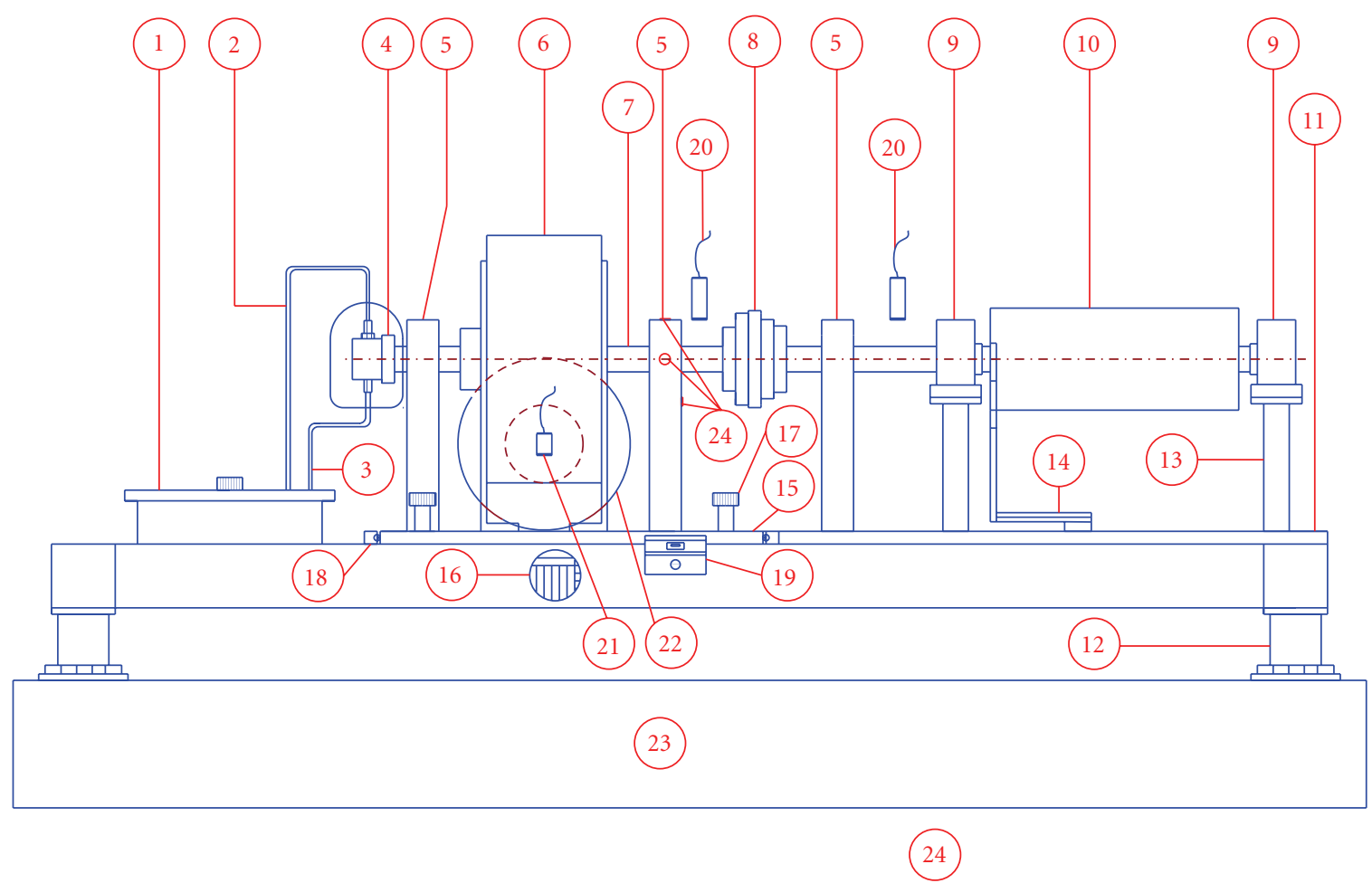

Figure 1: Centrifugal Blower Experimental Test Rig. 1, Oil sump fitted with motor; 2, Oil entry hose pipe; 3, Oil exit hose pipe; 4, Journal bearing; 5, Z-Type Ball Bearings; 6, Forward Curved Centrifugal Blower; 7, Shaft; 8, Magnetic coupling; 9, Self-aligning P Block Bearings; 10, Variable Speed DC motor; 11, Supporting structure; 12, Clamping screws; 13, Motor supporting frame; 14, Load cell; 15, Misaligning adjusting steel plate; 16, Plate movement screw (lead screw attachment is not shown); 17, Four screws for vertical direction adjustment of steel plate; 18, Two leveling support bars; 19, Digital Vernier caliper; 20, Proximity switches; 21, Hotwire Anemometer; 22, Butterfly Valve; 23, Concrete base; and 24, Locations of Accelerometer.

TABLE 1: The technical specifications of forward curved centrifugal blower.

\begin{tabular}{|c|c|c|c|}
\hline Name & Symbols & Unit & Value \\
\hline Outer diameter & $d_{2}$ & $\mathrm{~mm}$ & 135 \\
\hline Inner diameter & $d_{1}$ & $\mathrm{~mm}$ & 110 \\
\hline Number of blades & $N$ & & 36 \\
\hline Chord length & $l$ & $\mathrm{~mm}$ & 25 \\
\hline Blade width & $B$ & $\mathrm{~mm}$ & 71 \\
\hline Blade thickness & $T$ & $\mathrm{~mm}$ & 1.3 \\
\hline Blade inlet angle at the leading edge & $\beta_{1}$ & degrees & 112 \\
\hline Blade outlet angle at the tip of the blade & $\beta_{2}$ & degrees & 129 \\
\hline Blade channel width & $w$ & $\mathrm{~mm}$ & 10.20 \\
\hline Diameter ratio & $d_{1} / d_{2}$ & & 0.815 \\
\hline Blower end exit duct area & $A$ & $\mathrm{~m}^{2}$ & 0.00295 \\
\hline Weight of blower & $W$ & $\mathrm{~kg}$ & 2 \\
\hline
\end{tabular}

the power supply to the motor to enable the motor speed and blower speed to be held at specific cutoff speeds for testing. A control switch is used to engage the blower shaft and motor shaft by energizing the electromagnetic coupling. An instrumentation control panel is built in to display and control the variables.

A software developed using Visual Basic is used along with instrumentation to control the operation of experimental test rig and to record the motor and blower speeds as well as CDT for each test run for selected cutoff speeds. At the start of test run, the system automatically cuts off the power supply to the motor and electromagnetic coupling simultaneously so that the blower shaft completely disengages from motor shaft. At the end of the test, the power supply is restored to both motor and coupling so that they run continuously. Software has an ability to record CDT with an accuracy of $0.06 \mathrm{~s}$ ( $60 \mathrm{~ms})$ intervals, and corresponding deceleration speed of blower and motor at each test run is 
saved in a data file. The same can be exported to excel file for further analysis.

The LabVIEW7 [33, 34] application software is developed by the author for FFT analyzer and used to acquire vibration signals data via four-channel sensor input module Data Acquisition Device (NI-DAQ-National InstrumentsNI SCXI-1000 chassis via SCXI-1530-channel 0, SCXI-1530channel 1 and SCXI-1530-channel 2). Three piezoelectric accelerometers (Model 600A12, IMI sensors, frequency range up to $18 \mathrm{kHz}$ ) were used to acquire vibrations signals in vertical, horizontal, and axial directions. Two self designed fixtures are fitted to bearing housings at both blower end and motor end to hold the accelerometers in horizontal and axial direction. The LabVIEW7 application software displays the vibration spectrum over a range of frequencies and time scale. Vibration data can be exported as data file for further processing using MATLAB software package.

\section{Experimental Procedure}

The main objective of this research study is to explore the use of CDT analysis to detect and analyze the effect of misalignment and unbalance in forward curved centrifugal blower rotating machine. The blower shaft and motor shaft are carefully aligned and balanced in both vertical and horizontal directions using reverse dial indicator method. An electromagnetic coupling was used to ensure that the entire centrifugal blower system is completely free from the power source during coast down period test run. This is to minimize the effect of external disturbance due to the fluctuation in power source voltage, frequency, and co on that can have an appreciable effect on CDT. Initially, the coast down times for each test run at various cutoff speeds were recorded and used thereafter as baseline references for further investigation, analysis, and comparison. The baseline CDT obtained for blower shaft at cutoff speeds of $1000 \mathrm{rpm}$, $1500 \mathrm{rpm}, 2000 \mathrm{rpm}$, and $2500 \mathrm{rpm}$ are $1560 \mathrm{~ms}, 2040 \mathrm{~ms}$, $2580 \mathrm{~ms}$, and $3000 \mathrm{~ms}$, respectively under healthy operating conditions. The baseline CDT profiles for healthy operating condition at different blower shaft cutoff speeds are depicted in Figure 2. The vibration signatures for balanced and aligned condition at blower end bearing housing at all selected cutoff speeds in all the three direction were recorded. A minute amount of misalignment and residual imbalance are noticed in the spectrum. The amplitude level of vibration at $1 \mathrm{X}, 2 \mathrm{X}$ and $3 \mathrm{X}$ components are $0.2122 \mathrm{~m} / \mathrm{sec}^{2}$, $0.3315 \mathrm{~m} / \mathrm{sec}^{2}$, and $0.1355 \mathrm{~m} / \mathrm{sec}^{2}$, respectively, These values are well within the acceptable tolerance limits for the system. These vibration signatures are used as baseline signatures for the study and for analyzing the various mechanical faults.

Parallel misalignment, combined both in horizontal and vertical direction, is introduced between the blower shaft and motor shaft to study and analyze the effect in FC centrifugal blower and to understand the behavior of the system under these conditions. By loosening four screws and rotating the knob attached to the lead screw in clockwise direction, the blower setup steel plate is moved in horizontal direction away from the operator side to get the desired offset distance between the blower shaft and motor shaft

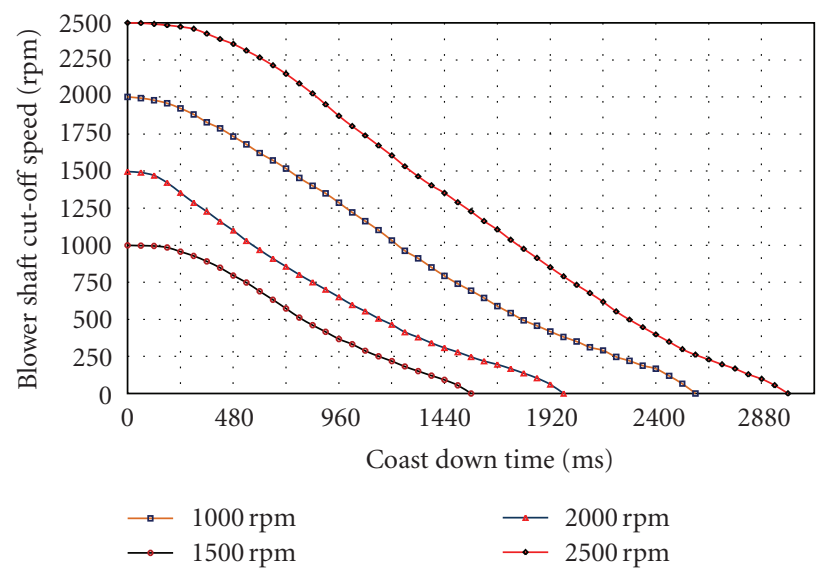

FIgURE 2: CDT profiles for healthy condition.

in horizontal direction. The possible movement of the steel plate in other directions was restricted by means of level bars supported with steel balls support. The created offset distance was measured by digital Vernier Caliper which is attached to the steel base plate. Holding the base steel plate in this position, vertical parallel misalignment was created by adjusting the gap between the blower setup steel plate and the main steel support frame by adjusting all the four screws in upward direction and inserting shims of specific thickness between the gaps on all four sides to lift it upward to create the required vertical offset distance with respect to the main frame. Three levels of combined offset misalignment distances of $0.10 \mathrm{~mm}, 0.20 \mathrm{~mm}$, and $0.30 \mathrm{~mm}$, respectively, have been introduced at the blower end shaft both in horizontal and vertical directions. The coast down time and the respective deceleration speeds were recorded.

Combination of parallel misalignment in horizontal direction with angular misalignment was created between the blower shaft and motor shaft. The misalignment angle was created towards the blower side shaft. Horizontal parallel misalignment was introduced with offset distances of $0.15 \mathrm{~mm}, 0.25 \mathrm{~mm}$, and $0.35 \mathrm{~mm}$, respectively. Angular misalignment was introduced by creating a gap between the steel plate and main support frame by adjusting the screws in one direction and inserting shims of specific thickness between the gaps to lift it upward with respect to the main frame, and then the corresponding inclined misaligned angle was calculated. Three levels of angular misalignment $0.046^{\circ}, 0.076^{\circ}$, and $0.107^{\circ}$, respectively, have been introduced between blower and motor shaft end.

Three cases of unbalance conditions were created by adding additional masses of 25 gram-mm, 30 gram-mm and 35 gram-mm, respectively on blower impeller blade. The masses have been added on blower impeller blade at the same location for every test.

For all the above three faulty cases, experimental tests were conducted at blower shaft cutoff speeds of $1000 \mathrm{rpm}$, $1500 \mathrm{rpm}, 2000 \mathrm{rpm}$, and $2500 \mathrm{rpm}$, respectively to record coast down time and the respective deceleration speed of blower shaft. Each experiment was repeated 5 times for 


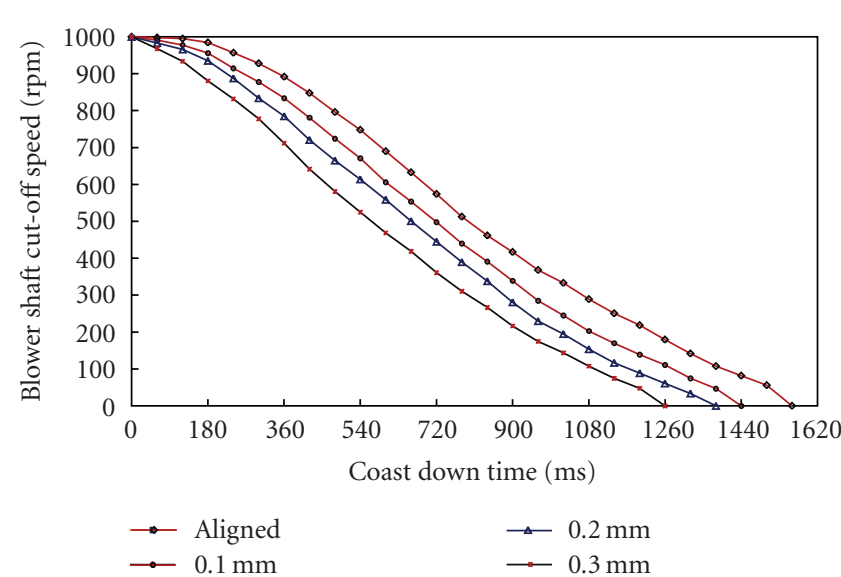

(a)

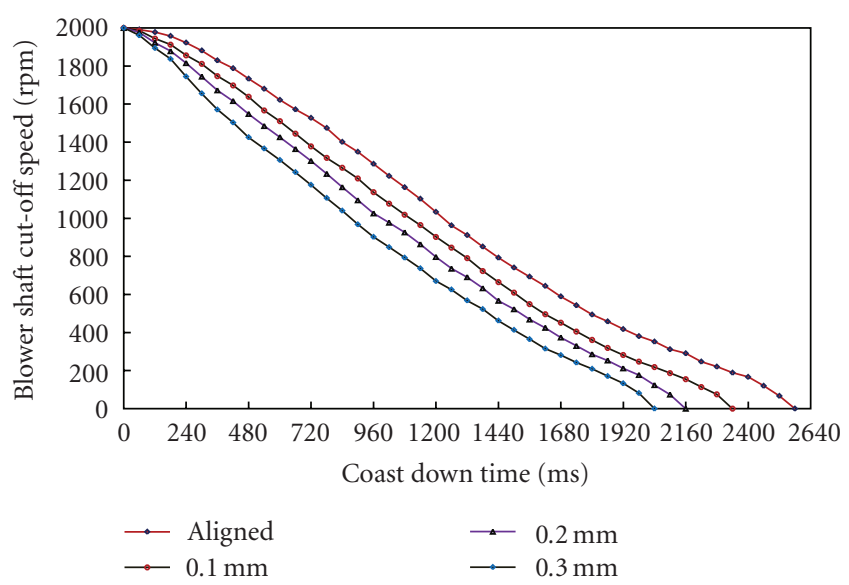

(c)

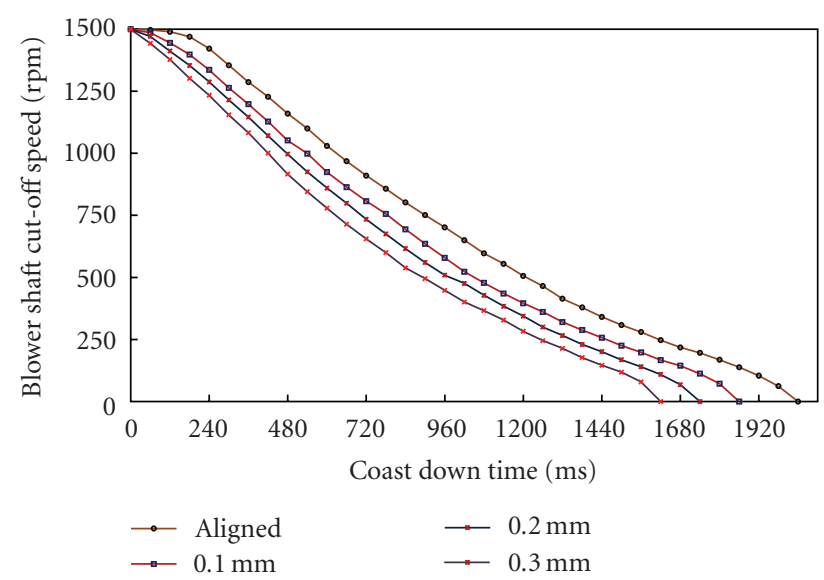

(b)

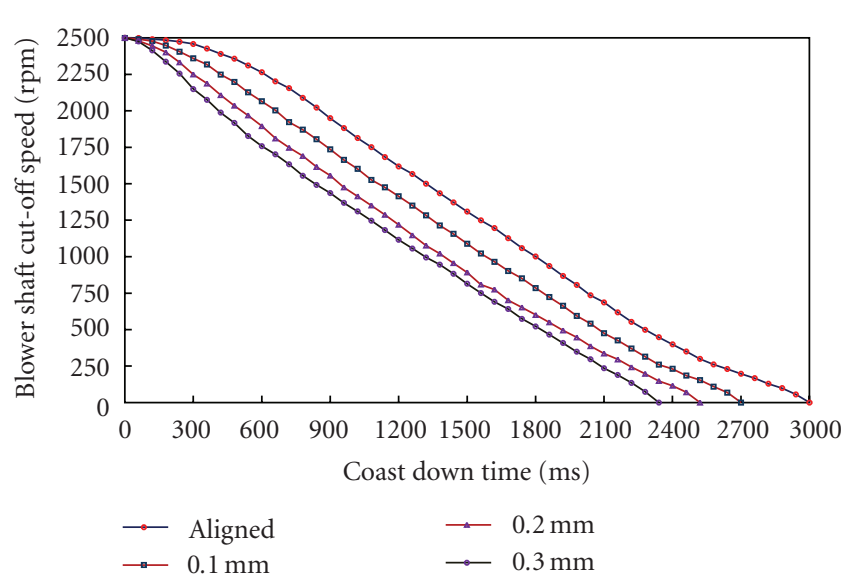

(d)

FIGURE 3: CDT profiles for different combined horizontal and vertical parallel misalignment conditions at cutoff speed of (a) $1000 \mathrm{rpm}$, (b) $1500 \mathrm{rpm}$, (c) $2000 \mathrm{rpm}$, and (d) $2500 \mathrm{rpm}$.

consistency under the same operating conditions to record CDTs. To verify the validity and accuracy of CDT data error analysis was carried out for sample size of 5, it was ascertained that the magnitude of the error is less than 1 percent in all the cases of both healthy and fault conditions. The repeatability of CDT data is around 99 percent.

Vibration data was acquired at blower end shaft bearing support housing in vertical, horizontal and axial directions. The sampling frequency used for data acquisition was $16 \mathrm{kHz}$ and the numbers of sampled data are 38400 with sampling time being $2.4 \mathrm{~s}$. Accelerometers are maintained in the same location position in all the three axes for each test run to acquire vibration data for consistency. The repeatability of vibration data is almost consistent in all the three directions.

\section{Results and Discussions}

The purpose of introducing the various levels of combined horizontal and vertical parallel misalignment, combined horizontal parallel and angular misalignment, and the different unbalance conditions is to study the mechanical behavior of the system during these conditions at different blower shaft cutoff speeds. Consequently, the influence of these mechanical fault conditions on CDT values is noticed and compared with respect to vibration spectrums. The CDT profiles and the speed in rpm versus CDT in milliseconds with a time interval of 60 milliseconds for balanced and aligned condition for combined horizontal and vertical parallel misalignment at offset distances of $0.10 \mathrm{~mm}$, $0.20 \mathrm{~mm}$, and $0.30 \mathrm{~mm}$, respectively in both directions at the blower shaft side are shown in Figure 3.

The typical CDT curves are characterized by three zones, at the beginning of the coast down as a small convex shape, at the middle of the coast down as a concave shape, and at the end of the coast down as a small convex shape. It has been observed that the slopes of the curves vary slightly from one another. Higher energy dissipation takes place during the middle of the coast down with the decrease in rotational speed. This is only due to fluid friction which decreases with the decrease in speed. At the end of the coast down, a small convex shape is due to metal-to-metal contact; the resistance to movement increases with decrease in rotational speed. At higher speeds, the CDT profile curves are much sharper and smooth when compared at lower speeds and these 


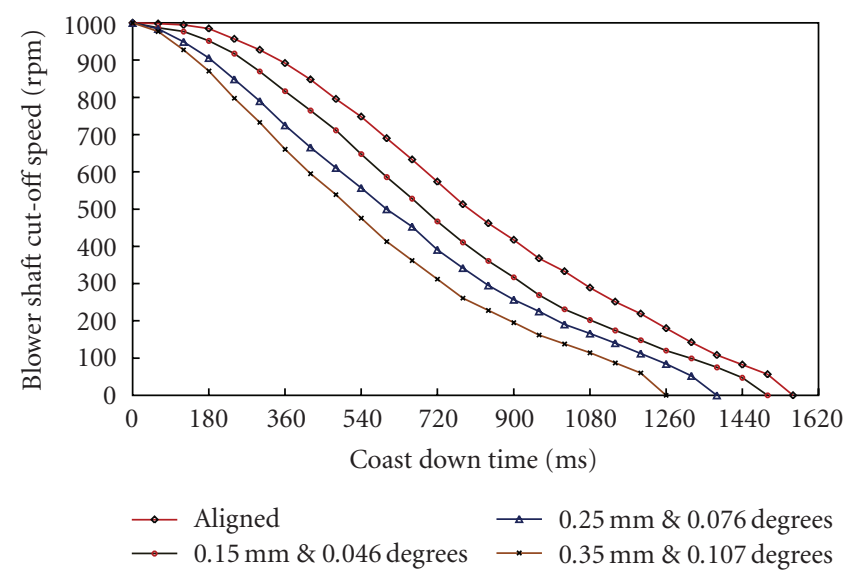

(a)

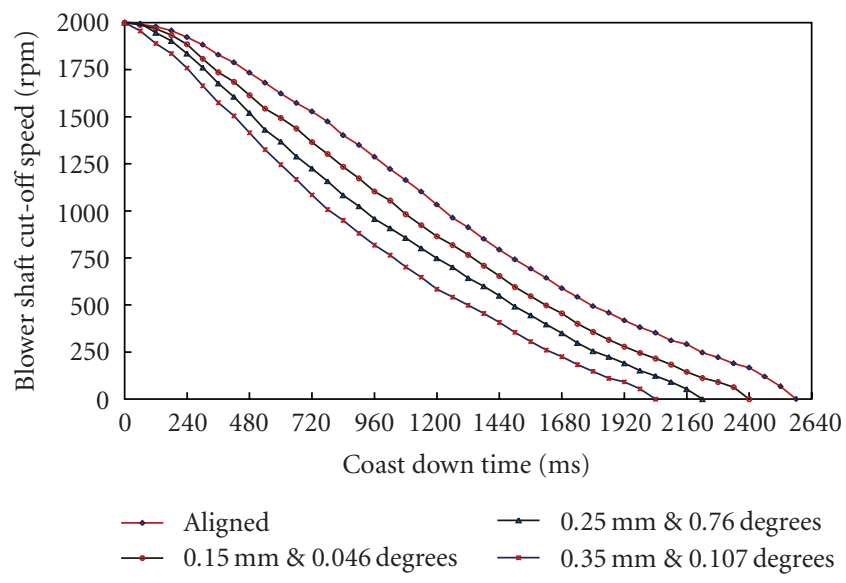

(c)

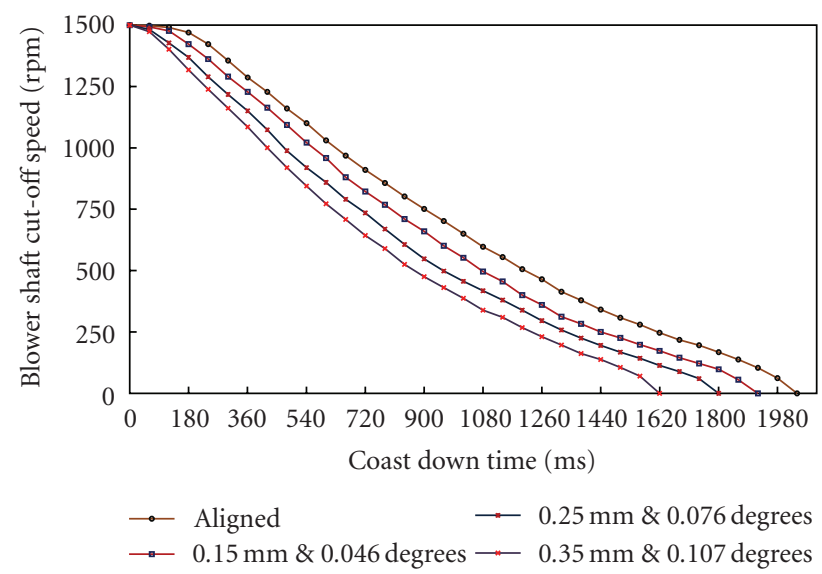

(b)

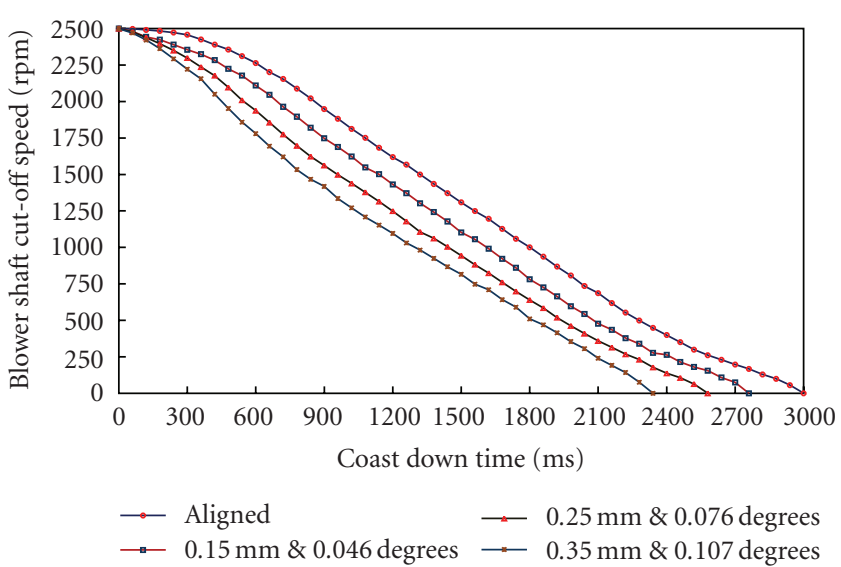

(d)

FIGURE 4: CDT profiles for different combined parallel and angular misalignment conditions at cutoff speed of (a) $1000 \mathrm{rpm}$, (b) $1500 \mathrm{rpm}$, (c) $2000 \mathrm{rpm}$, and (d) $2500 \mathrm{rpm}$.

profile curves follow the frictional characteristic described by Raimondi and Boyd design curve [35]. It was found that as malfunctions progresse, the blower shaft comes to rest faster within a lesser time. This is due to the increased power loss and increased torque in the bearings which is once again due to increased malfunction. Since the blower shaft is completely free from the driving shaft during the coast down period, as predicted, the blower shaft takes a longer time to dissipate the acquired energy during sustainable operation at higher running speeds. Consequently a higher CDT is obtained.

The profile curves for aligned condition and intentionally introduced combination of horizontal parallel and angular misalignment with offset distances in horizontal direction and inclined angles between blower shaft and motor shaft towards blower shaft end are $\left(0.15 \mathrm{~mm}, 0.046^{0}\right),(0.25 \mathrm{~mm}$, $\left.0.076^{\circ}\right)$, and $\left(0.35 \mathrm{~mm}, 0.107^{\circ}\right)$, respectively which are shown in Figure 4. It has been observed that at lower speed the effect on CDT is very less when compared to higher speed. An increase in vibration amplitude has been observed at higher cutoff speeds. By analyzing the trend of the CDT behavior during the deceleration period for various order of misalignment at different cutoff speeds, the behavior of the rotating system could be analyzed and also used as a diagnostic tool for condition monitoring of the rotating systems.

The CDT profile for balanced condition and intentionally introduced unbalance condition of masses of 25 gram$\mathrm{mm}, 30$ gram- $\mathrm{mm}$, and $35 \mathrm{gram}-\mathrm{mm}$ on the blower impeller blades at cutoff speeds of $1000 \mathrm{rpm}, 1500 \mathrm{rpm}, 2000 \mathrm{rpm}$, and $2500 \mathrm{rpm}$, respectively, are shown in Figure 5.

As the unbalance weights increased, with an increase in cut-off speeds, the unbalance forces on the blower impeller blades showed an increase, which in turn caused the CDT profile to be lowered. This deviation between the CDT profiles clearly indicates the influence of the unbalanced forces on the blower impeller. As unbalance weight increases, the blower shaft comes to rest faster in less time, which emphasizes that the mechanical behavior has considerable influence on the recorded CDT values.

The CDT parameter is a direct measure of the total friction in a rotating system. The results show that with increase in level of combined parallel, misalignment, combined parallel and angular misalignment, and with increase in unbalance 


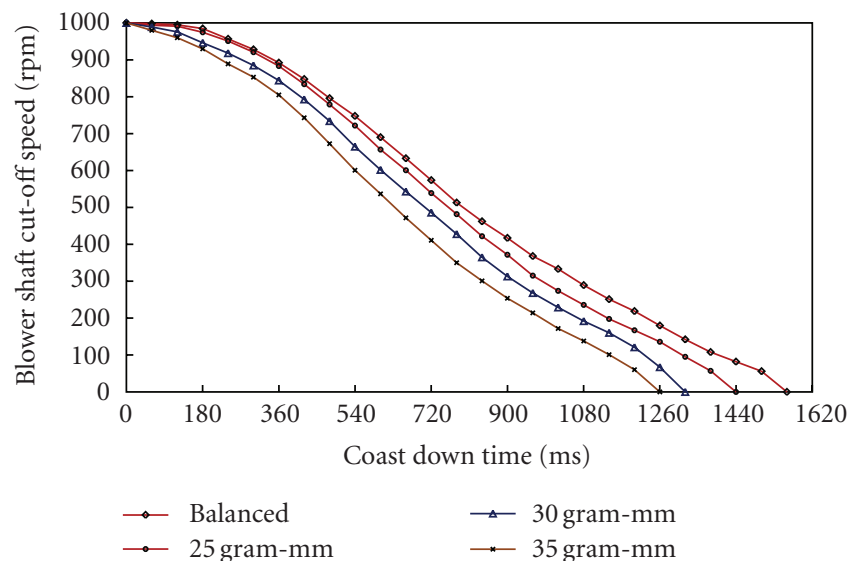

(a)

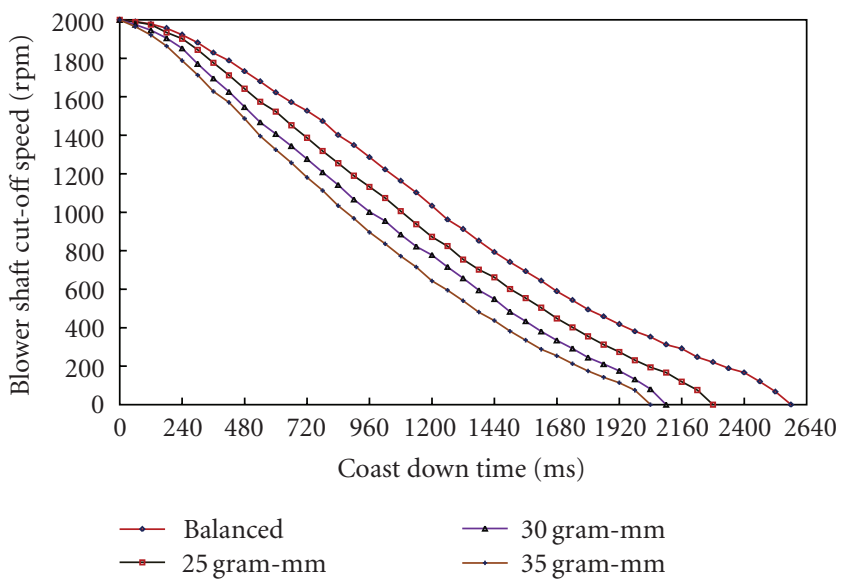

(c)

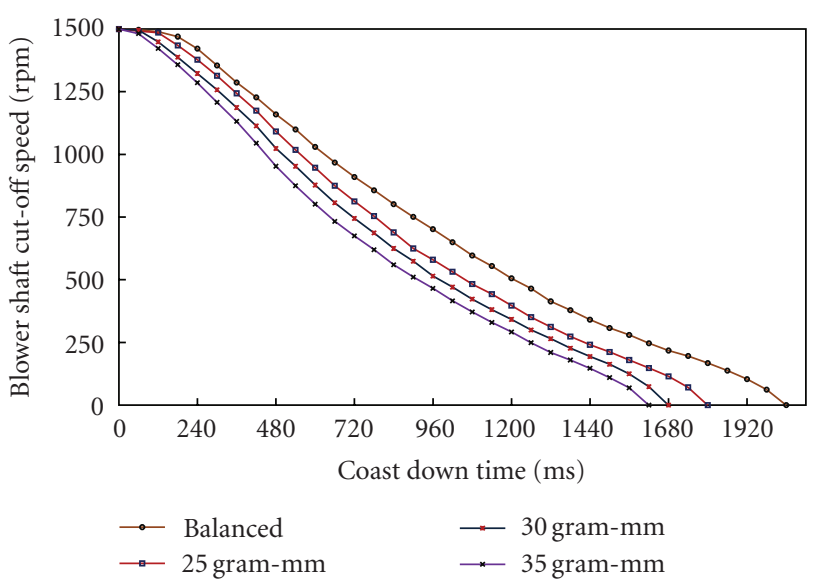

(b)

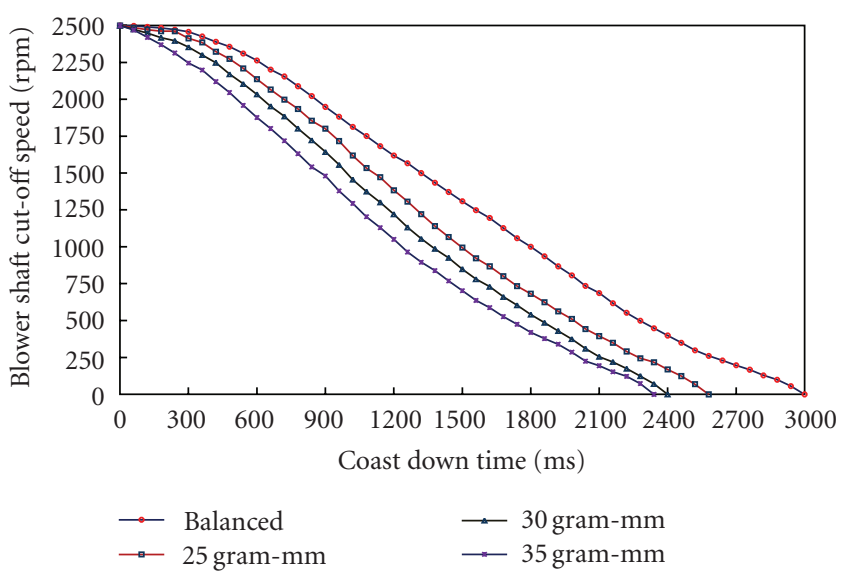

(d)

Figure 5: CDT profiles for different unbalance conditions at cutoff speed of (a) $1000 \mathrm{rpm}$, (b) $1500 \mathrm{rpm}$, (c) $2000 \mathrm{rpm}$, and (d) $2500 \mathrm{rpm}$.

weight, the coast down time decreases compared to baseline CDT recorded under normal operating conditions. At lower cutoff speed, the profiles of the CDT curve are more concave shape than at higher cutoff speeds.

The vibration spectrums of frequency domain for the blower shaft running at the speed of $2500 \mathrm{rpm}(41.667 \mathrm{~Hz})$ for different levels of combined horizontal and vertical parallel misalignment are presented in Figure 6. Similarly, the vibration spectrums for various orders of combined horizontal parallel misalignment and angular misalignment are presented in Figure 7. In both cases, the vibration spectrums in axial direction are presented. For different unbalance conditions, the vibration spectrums along the horizontal direction are shown in Figure 8. Vibration spectrums at higher blower shaft speed of $2500 \mathrm{rpm}$ are presented here for discussion to highlight the effect of mechanical malfunctions. In all the three vibration spectrums, the top figure shows the zoomed spectrum for balanced and aligned conditions. The figures followed below the top figure show the frequency spectrums in the order of introduced combined horizontal and vertical parallel misalignment, combined horizontal parallel and angular misalignment, and different unbalance conditions. The magnitude of vibration indicates the particular faults observed.

As in Figure 6, the high vibration amplitude is observed in axial direction than radial direction. It has been observed that the vibration amplitude in axial direction is more than $50 \%$ of the radial vibration amplitude; it clearly indicates the presence of misalignment. In vibration spectrums of frequency domain for various levels of combined horizontal and vertical parallel misalignment, it is observed that the $2 \mathrm{X}$ $(83.33 \mathrm{~Hz})$ running speed vibration amplitude component is the predominant frequency in the spectrum in all the three faulty cases. It is found that the $2 \mathrm{X}$ component for $0.10 \mathrm{~mm}$ offset is $1.134861 \mathrm{~m} / \mathrm{sec}^{2}$ and ncreases to $1.628757 \mathrm{~m} / \mathrm{sec}^{2}$ as offset misalignment increases to $0.30 \mathrm{~mm}$. This agrees with the fact that misalignment causes an increase in $2 \mathrm{X}$ amplitude. This $2 \mathrm{X}$ component increases as the order of offset misalignment increases; also vibration amplitude is a function of operating running speed and consequently has the influence on CDT and on behavior of the system. A small increase in 1X amplitude level is also observed, which is because of centrifugal force effect due to residual 


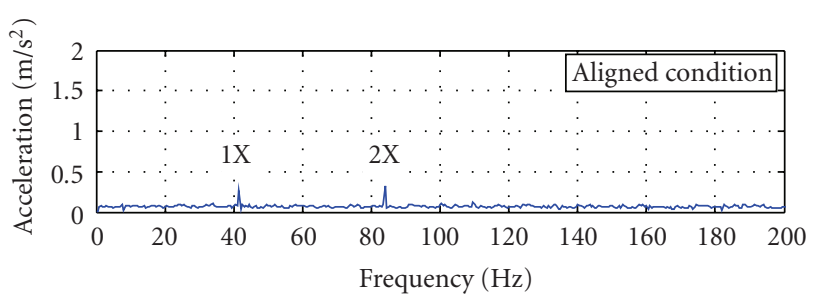

(a)

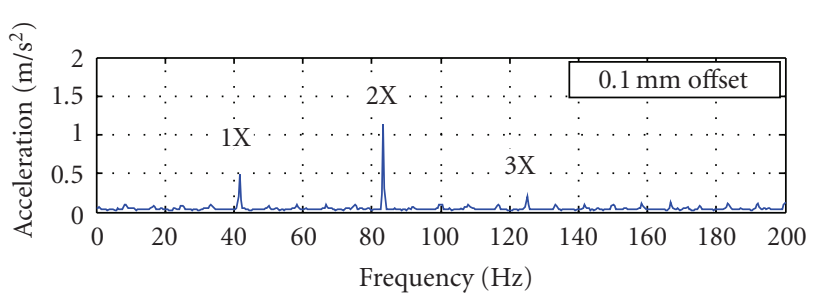

(b)

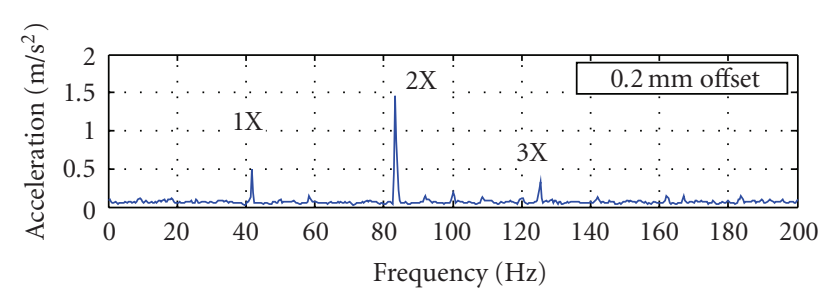

(c)

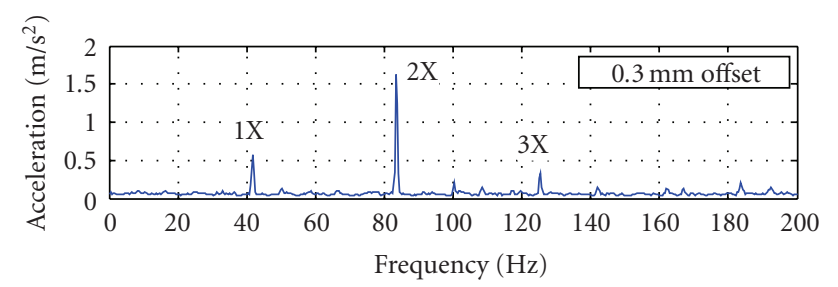

(d)

Figure 6: Vibration spectrums for combined horizontal and vertical parallel misalignment.

unbalance. For increased misalignment, vibrations at multiple harmonics are evident due to mechanical behavior of the system, and also due to the strain induced in the shaft and because of interaction between the impeller blades and the air drag force. It is observed that the vibration amplitude level increases as operating speed increases and with increase in offset misalignment. There is a drastic increase in the vibration amplitude level as order of offset misalignment increases.

As seen in Figure 7, the vibration spectrums of frequency domain for various orders of combined horizontal parallel misalignment and angular misalignment, it is observed that the $2 \mathrm{X}(83.33 \mathrm{~Hz})$ running speed vibration amplitude component is the predominant frequency in the spectrum in all the three faulty cases. And also it is noticed that the $3 \mathrm{X}(125 \mathrm{~Hz})$ running speed vibration amplitude components also increase as combined misalignment increases. It is found that the $2 \mathrm{X}$ component for $\left(0.15 \mathrm{~mm}, 0.046^{0}\right)$ is

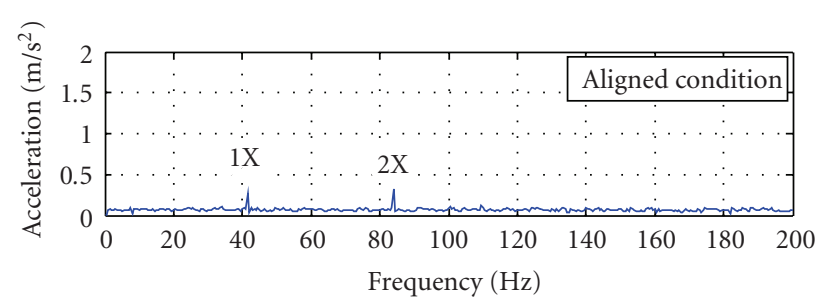

(a)

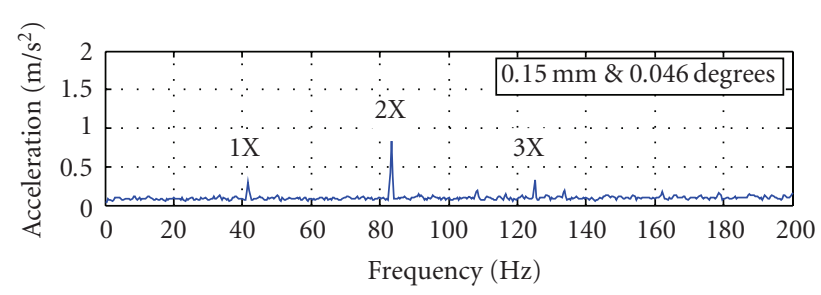

(b)

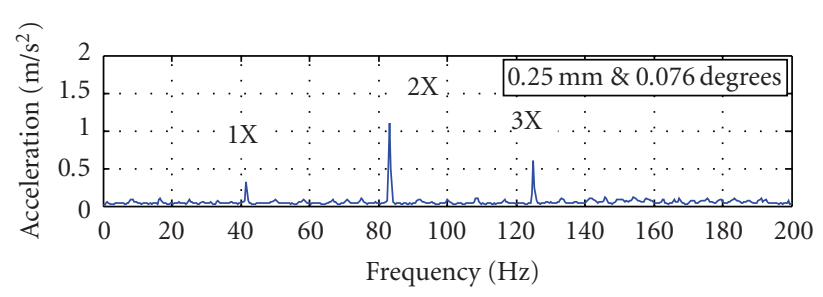

(c)

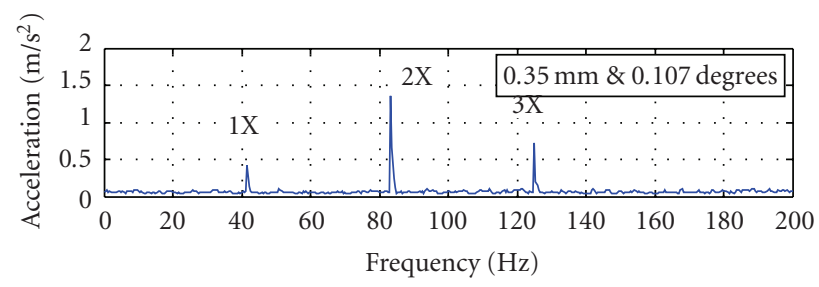

(d)

FIGURE 7: Vibration spectrums for combined parallel misalignment and angular misalignment.

$0.830513 \mathrm{~m} / \mathrm{sec}^{2}$ and increases to $1.359472 \mathrm{~m} / \mathrm{sec}^{2}$ as combined misalignment increases to $\left(0.35 \mathrm{~mm}, 0.107^{0}\right)$. This clearly indicates the presence of combined misalignment, and the amplitude increases as the order of combined misalignment increases; also vibration amplitude is a function of operating running speed and consequently has the influence on CDT and on the behavior of the system. Slight increase in $1 \mathrm{X}$ amplitude level is also observed, which is because of centrifugal force effect due to residual unbalance. And, also due to combined misalignment, more strain is induced in the coupling, which causes increased values of vibration amplitude at $2 \mathrm{X}$ and $3 \mathrm{X}$ shaft running speed. For increased misalignment, vibrations at multiple harmonics are evident due to mechanical behavior of the system, and also due to the strain induced in the shaft and because of interaction between the impeller blades and the air drag force. There is a drastic increase in the vibration amplitude level as the order of combined misalignment increases. 


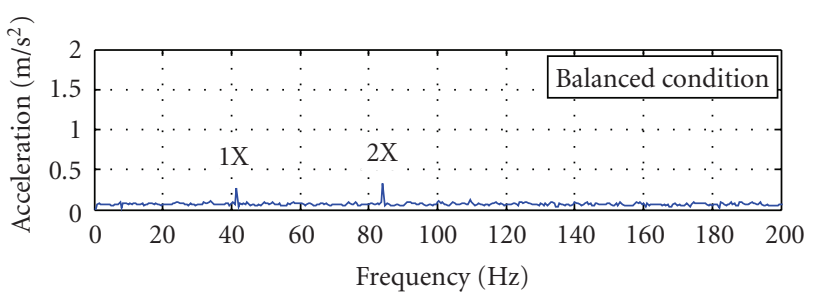

(a)



(b)

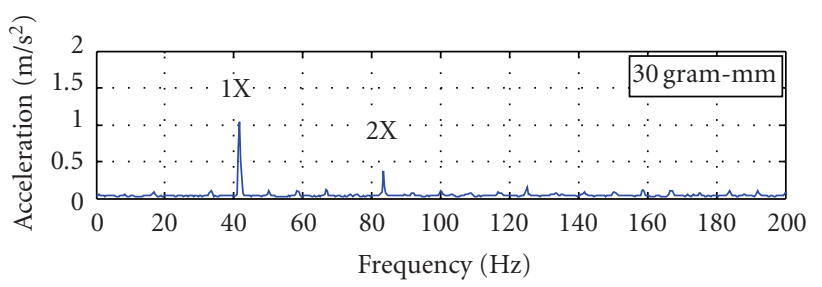

(c)

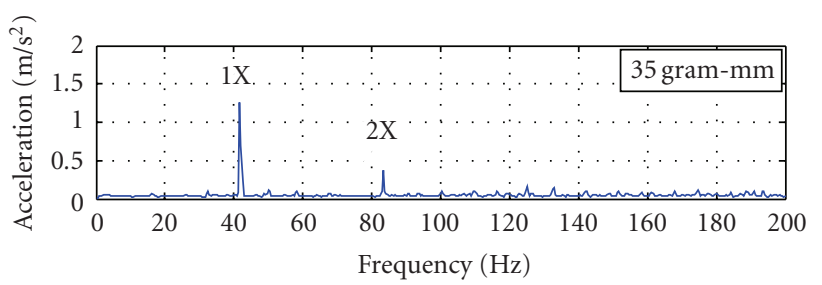

(d)

FIgURE 8: Vibration spectrums for different unbalance condition.

Figure 8 shows the high vibration amplitude observed in horizontal direction than in axial direction, clearly indicating the presence of unbalance. The vibration frequency of rotating system unbalance is synchronous, that is, one time the shaft speed $(1 \mathrm{X})$, since the unbalance force rotates at the shaft running speed. For different unbalance conditions, it is observed that the $1 \mathrm{X}(41.667 \mathrm{~Hz})$ running speed component is the predominant frequency in the spectrum in all the three faulty cases. This clearly shows the presence of unbalance and that the vibration amplitude increases as the unbalance weight increases and also the vibration amplitude is a function of operating running speed. It is found that the $1 \mathrm{X}$ component for 25 gram-mm weight is $0.823564 \mathrm{~m} / \mathrm{sec}^{2}$; as the weight increased to 30 and 35 grams, the respective $1 \mathrm{X}$ component increases to $1.034582 \mathrm{~m} / \mathrm{sec}^{2}$ and $1.256434 \mathrm{~m} / \mathrm{sec}^{2}$. From this it is evident that as the unbalance weight increases, it has a considerable influence on the behavior of the system and also on CDT valves.

The obtained CDT values for all the three introduced fault cases, corresponding to blower rotational speeds, are

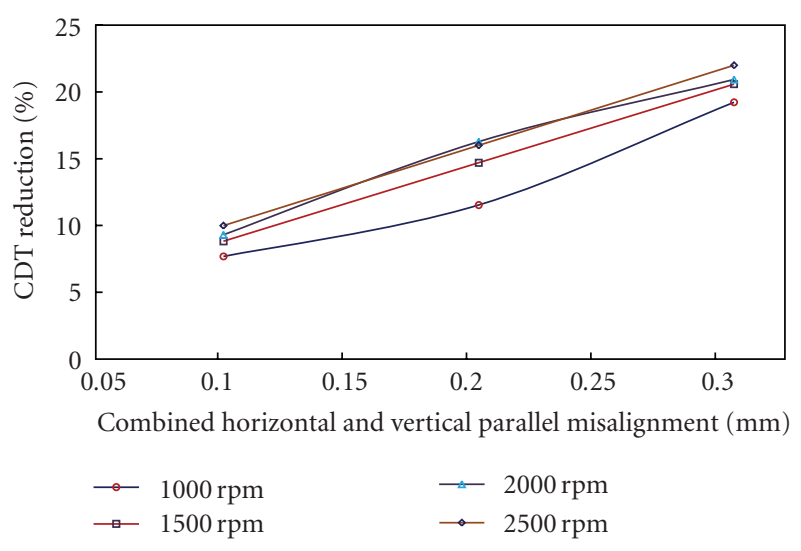

FIGURE 9: CDT reduction percentages for combined horizontal and vertical parallel misalignment.

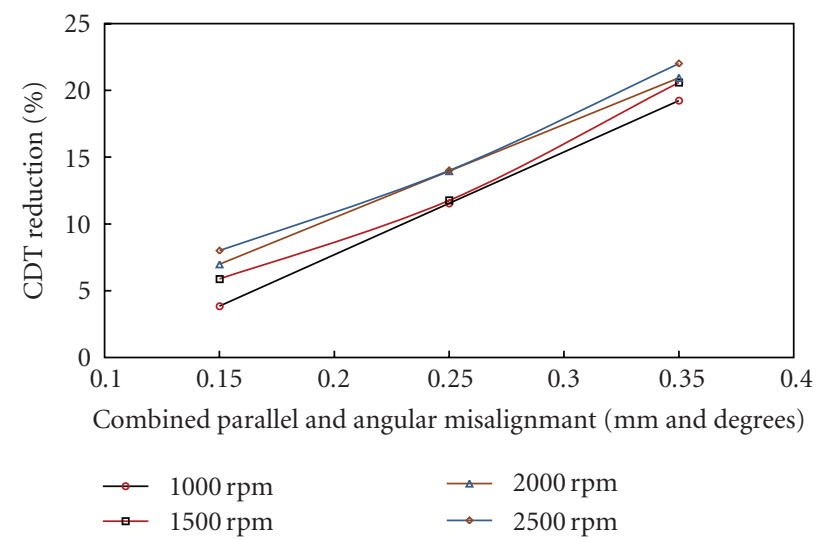

FIGURE 10: CDT reduction percentages for combined parallel and angular misalignment.

depicted as CDT reduction percentages versus combined horizontal and vertical parallel misalignment, combined horizontal parallel and angular misalignment, and for various unbalance conditions are represented in Figures 9, 10 and 11 , respectively. The CDT reduction percentage is calculated using the relation,

$$
\begin{aligned}
& \text { CDT reduction percentage } \\
& \qquad=\left[\frac{\text { baseline CDT }- \text { obtained CDT }}{\text { baseline CDT }}\right]^{*} 100 .
\end{aligned}
$$

In all the three cases, it is observed that high increase in CDT reduction percentage correlates with increase in offset distance, angle of angular misalignment and unbalance weight. The impact on CDT reduction percentage is very less at lower cutoff speed, at smaller offset distances, at smaller order of angular misalignment, and at less unbalance weights. At higher order of misalignment and at high unbalance weights with higher cutoff speed, the impact on the percentage reduction is very high, and from these plots it is noticed that there is a specific correlation between the CDT reduction percentages and the order of mechanical malfunctions (combined parallel misalignment or combined 


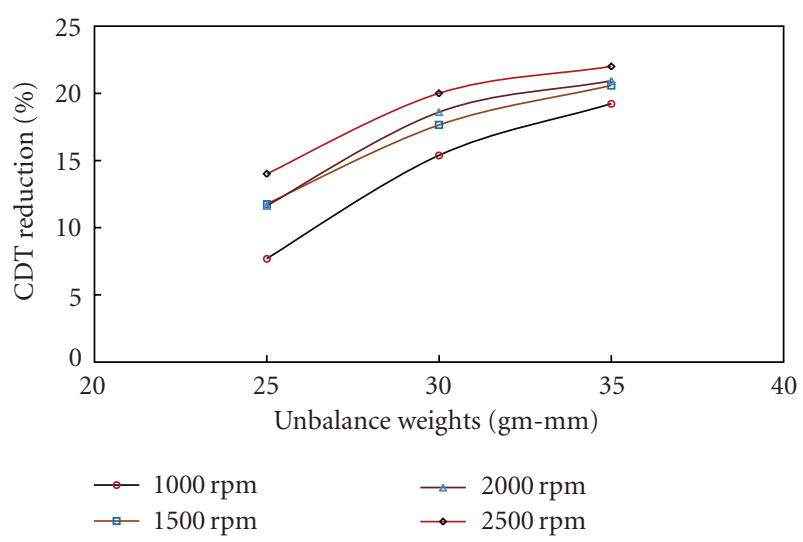

FIGURE 11: CDT reduction percentages for various unbalance conditions.



FIGURE 12: Torque for combined horizontal and vertical parallel misalignment.

parallel and angular misalignment and unbalance). It is worthwhile to mention here that as mechanical malfunction increases, the CDT reduction percentage gradually increases with increase in shaft rotational speed. It is also noted that the $\mathrm{CDT}$ and corresponding CDT reduction percentage have an effect on cutoff speed and are found to be changing in relation with the cutoff speed. And the mechanical malfunction has the considerable influence on CDT reduction percentage.

The driving torques before and after coupling were recorded under healthy conditions at cutoff speeds of $1000 \mathrm{rpm}, 1500 \mathrm{rpm}, 2000 \mathrm{rpm}$, and $2500 \mathrm{rpm}$, respectively. These torque values are compared with the corresponding measured torque for before and after coupling conditions for different combined horizontal and vertical parallel misalignment and various combined horizontal parallel and angular misalignment conditions and for different unbalance weights. The respective torque measurements for these conditions are shown in Figures 12, 13, and 14, respectively. The observed torque measurements indicate that as the misalignment increases in both cases of misalignment conditions, the torque after coupling also increases due to

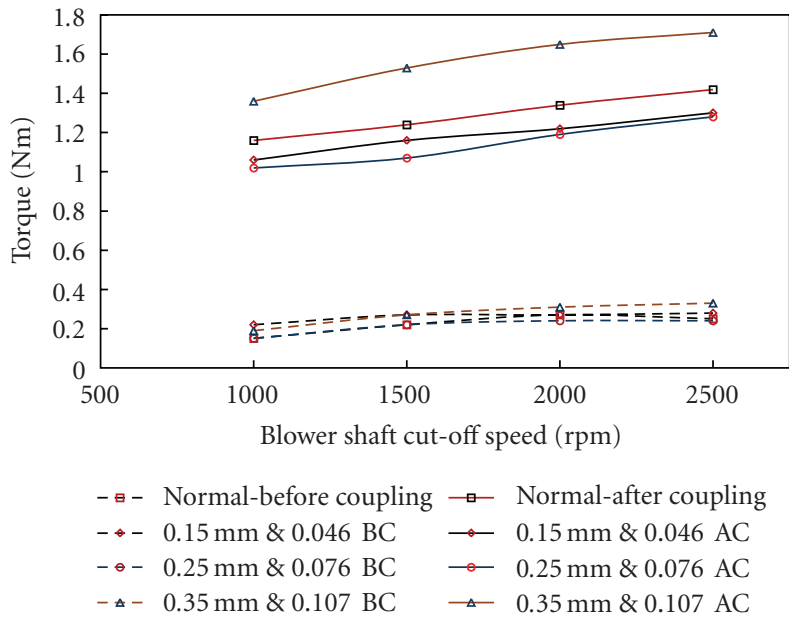

FIGURE 13: Torque for combined parallel and angular misalignment.

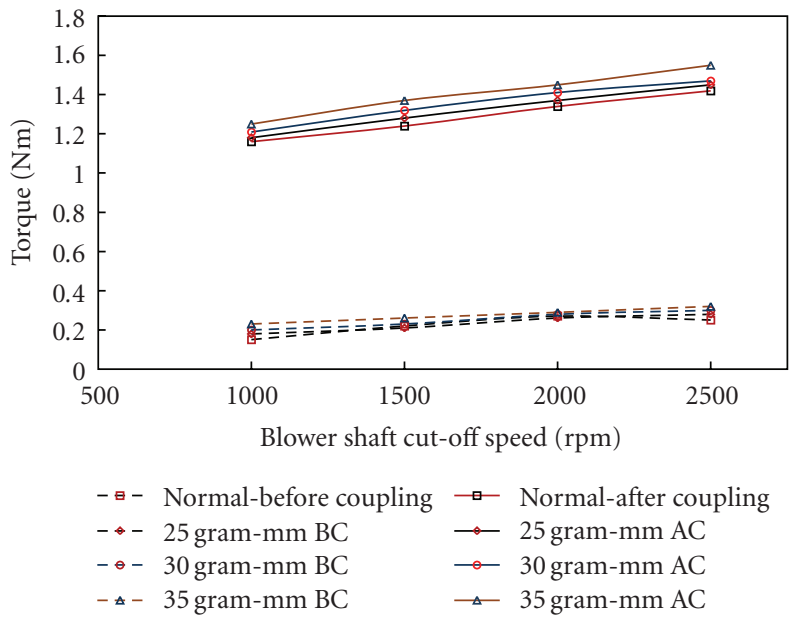

FIGURE 14: Torque for various unbalance conditions.

load on the coupling. A similar trend is observed in various unbalance conditions. It is observed that at higher speed, with higher order of introduced combined misalignment conditions and unbalance condition, the frictional torque is higher than at that lower speeds.

The speed reductions before and after coupling were recorded under healthy conditions at cutoff speeds of $1000 \mathrm{rpm}, 1500 \mathrm{rpm}, 2000 \mathrm{rpm}$, and $2500 \mathrm{rpm}$, respectively. These speed reduction values are compared with the corresponding measured speed reduction before and after coupling condition for different combined horizontal and vertical parallel misalignment, combined horizontal parallel and angular misalignment conditions, and for various unbalance conditions. The respective speed reduction measurements for different created mechanical faults are depicted in Figures 15,16 , and 17 as speed reduction percentages. It has been found that the speed reduction percentage decreases as rotational speed increases with tha increase in mechanical malfunctions. 


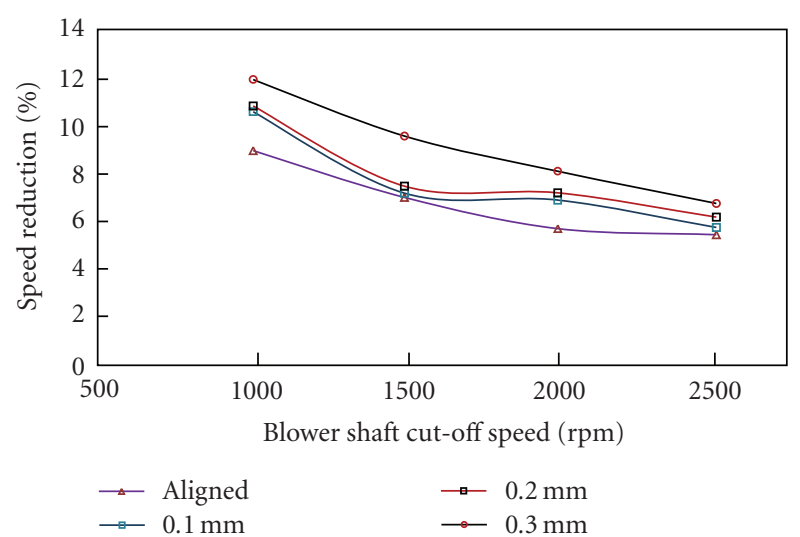

Figure 15: Speed reduction percentages for combined horizontal and vertical parallel misalignment..

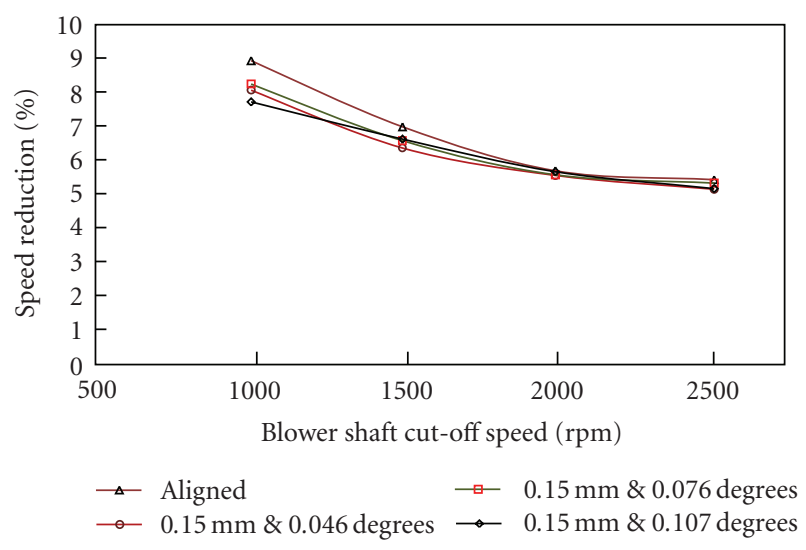

FIGURE 16: Speed reduction percentages for combined parallel and angular misalignment.

\section{Conclusion}

In this experimental investigation, the effects of combined horizontal and vertical parallel misalignment, combined horizontal parallel and angular misalignment, and unbalance conditions in rotating machinery are studied. It was found that the CDT decreases as mechanical malfunction increases. CDT reduction percentages were calculated for all three cases; it was observed that the CDT reduction percentage increases with the increase in mechanical malfunctions and with the increase in rotational speeds. There is a specific correlation between the CDT reduction percentage and the level of unbalance, the order of combined parallel misalignment, and the combined parallel and angular misalignment with rotational speed. The $2 \mathrm{X}$ vibration amplitude component is predominant with increase in combined offset misalignment. The $2 \mathrm{X}$ vibration amplitude component is predominant, also increases in $3 \mathrm{X}$ vibration component at higher combined parallel misalignment and angular misalignment with the increase in shaft rotational speed. As unbalance weight increases, the $1 \mathrm{X}$ vibration amplitude component increases considerably. The CDT decreases with increase in mechanical faults. The corresponding CDT reduction percentage

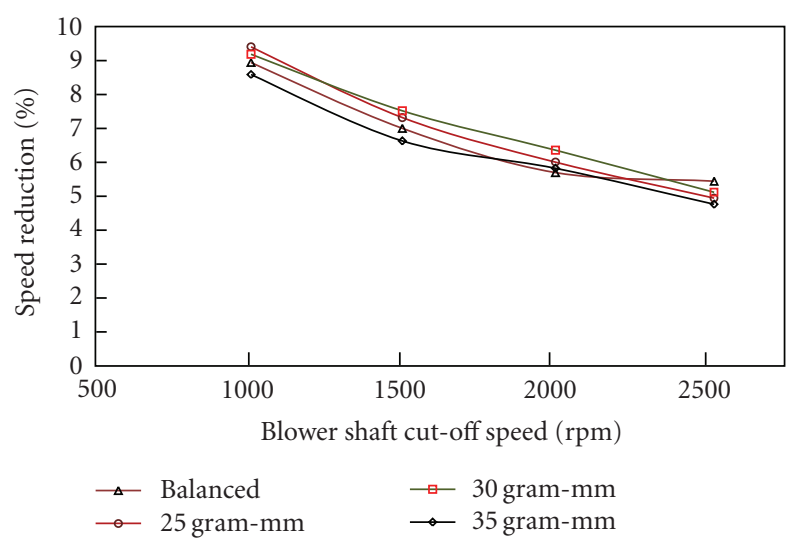

FIGURE 17: Speed reduction percentages for unbalance conditions.

increases with the increase in mechanical faults at higher rotational speeds. The frictional torque is higher than that at lower speeds with increased malfunctions. The speed reduction percentage decreases as rotational speed increases with the increase in mechanical malfunctions. This experimental investigation technique provides a simple method of evaluating the effect of mechanical faults in forward curved centrifugal blower using coast down time analysis and shows great potential to use this technique to predict mechanical malfunctions.

During the course of time, by frequent monitoring of the rotating system, recording the CDT values for selected operating speeds, and estimating the corresponding CDT reduction percentages, if any variations are observed between the baseline CDT and the obtained CDT values and the corresponding increase in CDT reduction percentage, one can detect, predict, and assess the severity of mechanical malfunction (misalignment and unbalance). The usual vibration analysis is performed along with CDT analysis to identify the particular mechanical malfunctions and find the root cause and further corrective action can be initiated to avoid serious damage and machinery failure. Hence, CDT analysis can be used as an effective diagnostic parameter for machine health monitoring.

\section{Acknowledgment}

G. R. Rameshkumar thankfully acknowledges the generous and continuous support of the Management, Principal, and Dean of the College for carrying out this research work for his Ph.D. under Staff Development.

\section{References}

[1] B. K. N. Rao, Handbook of Condition Monitoring, Elsevier Advanced Technology, Oxford, UK, 1st edition, 1996.

[2] D. E. Bently, Fundamentals of Rotating Machinery Diagnostics, Bently Pressurized Bearing Press, Minden, La, USA, 2002.

[3] P. John, Shaft Alignment Handbook, CRC Press; Taylor \& Francis Group LLC, New York, NY, USA, 3rd edition, 2007.

[4] K. P. Ramachandran, "Vibration signature analysis for machine health monitoring and fault diagnosis," Caledonian Journal of Engineering, pp. 26-39, 2004. 
[5] T. L. Daugherty and R. J. Craig, "Coast down time as a mechanical condition indicator," DTNSRDC Report, pp. 4547, 1976.

[6] T. L. Daugherty and R. J. Craig, "Coast down time as a mechanical condition indicator for vertical axis motors with grease-lubricated ball bearings," American Society of Lubrication Engineers Transactions, pp. 349-357, 1977.

[7] G. D. Xistris and D. C. Watson, "Using rundown time as bearing life indicator," Mechanical Engineering, vol. 98, no. 5, pp. 20-23, 1976.

[8] K. P. Ramachandran, M. Z. K. Malik, and A. Abdul Harees, "CDT analysis as a tool for evaluating bearing lubrication and mechanical conditions," Caledonian Journal of Engineering, pp. 19-24, 2004.

[9] R. E. Browne, K. P. Ramachandran, A. K. M. De Silva, and D. K. Harrison, "An experimental investigation to analyse the effect of unbalance in a horizontal rotor system using coastdown factor," International Journal of COMADEM, vol. 10, no. 3, pp. 11-18, 2007.

[10] B. S. Prabhu, "An experimental investigation on the misalignment effects in journal bearings," Tribology Transactions, vol. 40, no. 2, pp. 235-242, 1997.

[11] P. Arumugam, S. Swarnamani, and B. S. Prabhu, "Effects of journal misalignment on the performance characteristics of three-lobe bearings," Journal of Wear, vol. 206, no. 1-2, pp. 122-129, 1997.

[12] A. S. Sekhar and B. S. Prabhu, "Effects of coupling misalignment on vibrations of rotating machinery," Journal of Sound and Vibration, vol. 185, no. 4, pp. 655-671, 1995.

[13] G. Santhanakrishnan, B. S. Prabhu, and B. V. A. Rao, "An experimental investigation of tribological effects on coastdown phenomena in horizontal rotating machinery," Journal of Wear, vol. 91, no. 1, pp. 25-31, 1983.

[14] K. P. Ramachandran, M. Mahadevappa, M. K. Ravishankar, and A. Ramakrishna, "An approach to machine misalignment studies using vibration, orbit and coast down phenomena," in Proceedings of the International Conference on Advances in Mechanical and Industrial Engineering, University of Roorkee, Roorkee, India, 1997.

[15] S. Prabhakar, A. S. Sekhar, and A. R. Mohanty, "Vibration analysis of a misaligned rotor-coupling-bearing system passing through the critical speed," Proceedings of the Institution of Mechanical Engineers, Part C: Journal of Mechanical Engineering Science, vol. 215, no. 12, pp. 1417-1428, 2001.

[16] S. M. Huang, W. L. Chen, C. H. Yau, and C. K. Sung, "Effects of misalignment on the transmission characteristics of magnetic couplings," Proceedings of the Institution of Mechanical Engineers, Part C: Journal of Mechanical Engineering Science, vol. 215, no. 2, pp. 227-236, 2001.

[17] K. M. Al-Hussain and I. Redmond, "Dynamic response of two rotors connected by rigid mechanical coupling with parallel misalignment," Journal of Sound and Vibration, vol. 249, no. 3, pp. 483-498, 2002.

[18] K. M. Al-Hussain, "Dynamic stability of two rigid rotors connected by a flexible coupling with angular misalignment," Journal of Sound and Vibration, vol. 266, no. 2, pp. 217-234, 2003.

[19] D. L. Dewell and L. D. Mitchell, "Detection of a misaligned disk coupling using spectrum analysis," Transactions of the American Society of Mechanical Engineers, Journal of Vibration, Acoustics, Stress, and Reliability in Design, vol. 106, no. 1, pp. 9-15, 1984.

[20] M. Xu and R. D. Marangoni, "Vibration analysis of a motorflexible coupling-rotor system subject to misalignment and unbalance, part I: theoretical model and analysis," Journal of Sound and Vibration, vol. 176, no. 5, pp. 663-679, 1994.

[21] M. Xu and R. D. Marangoni, "Vibration analysis of a motorflexible coupling-rotor system subject to misalignment and unbalance, part II: experimental validation," Journal of Sound and Vibration, vol. 176, no. 5, pp. 681-691, 1994.

[22] P. N. Saavedra and D. E. Ramírez, "Vibration analysis of rotors for the identification of shaft misalignment part 1: theoretical analysis," Proceedings of the Institution of Mechanical Engineers, Part C: Journal of Mechanical Engineering Science, vol. 218, no. 9, pp. 971-985, 2004.

[23] P. N. Saavedra and D. E. Ramírez, "Vibration analysis of rotors for the identification of shaft misalignment part 2: experimental validation," Proceedings of the Institution of Mechanical Engineers, Part C: Journal of Mechanical Engineering Science, vol. 218, no. 9, pp. 987-999, 2004.

[24] J. K. Sinha, A. W. Lees, and M. I. Friswell, "Estimating unbalance and misalignment of a flexible rotating machine from a single run-down," Journal of Sound and Vibration, vol. 272, no. 3-5, pp. 967-989, 2004.

[25] M. A. Hili, T. Fakhfakh, and M. Haddar, "Failure analysis of a misaligned and unbalanced flexible rotor," Journal of Failure Analysis and Prevention, vol. 6, no. 4, pp. 73-82, 2006.

[26] Y.-S. Lee and C.-W. Lee, "Modelling and vibration analysis of misaligned rotor-ball bearing systems," Journal of Sound and Vibration, vol. 224, no. 1, pp. 17-32, 1999.

[27] M. A. Hili, T. Fakhfakh, L. Hammami, and M. Haddar, "Shaft misalignment effect on bearings dynamical behavior," International Journal of Advanced Manufacturing Technology, vol. 26, no. 5-6, pp. 615-622, 2005.

[28] A. W. Lee, "Some studies on misalignment in rigid coupling," in Proceedings of the 7th International Conference on Rotor Dynamics (IFTOMM '07), Vienna, Austria, 2007.

[29] A. W. Lees, "Misalignment in rigidly coupled rotors," Journal of Sound and Vibration, vol. 305, no. 1-2, pp. 261-271, 2007.

[30] A. Muszynska, "Vibrational diagnostics of rotating machinery malfunctions," International Journal of Rotating machinery, vol. 1, no. 3-4, pp. 237-266, 1995.

[31] F. B. Bleier, Fan Handbook Selection, Application, and Design, McGraw-Hill, London, UK, 1998.

[32] G. R. Rameshkumar, B. V. A. Rao, and K. P. Ramachandran, "An experimental investigation to study the effect of misalignment using CDT as a condition monitoring parameter for rotating machinery," in Proceedings of the 22nd International Congress of Condition Monitoring and Diagnostic Engineering Management ( COMADEM '09), pp. 531-539, San Sebastian, Spain, 2009.

[33] LabVIEW 7 Express, User Manual, National Instruments Corporate Headquarters, USA, 2003.

[34] LabVIEW 7 Express, User Manual, Measurements Instruments Corporate Headquarters, USA, 2003.

[35] J. S. Shighley and C. R. Mischke, Hand book of Machine Design, McGraw Hill, New York, NY, USA, 1976. 

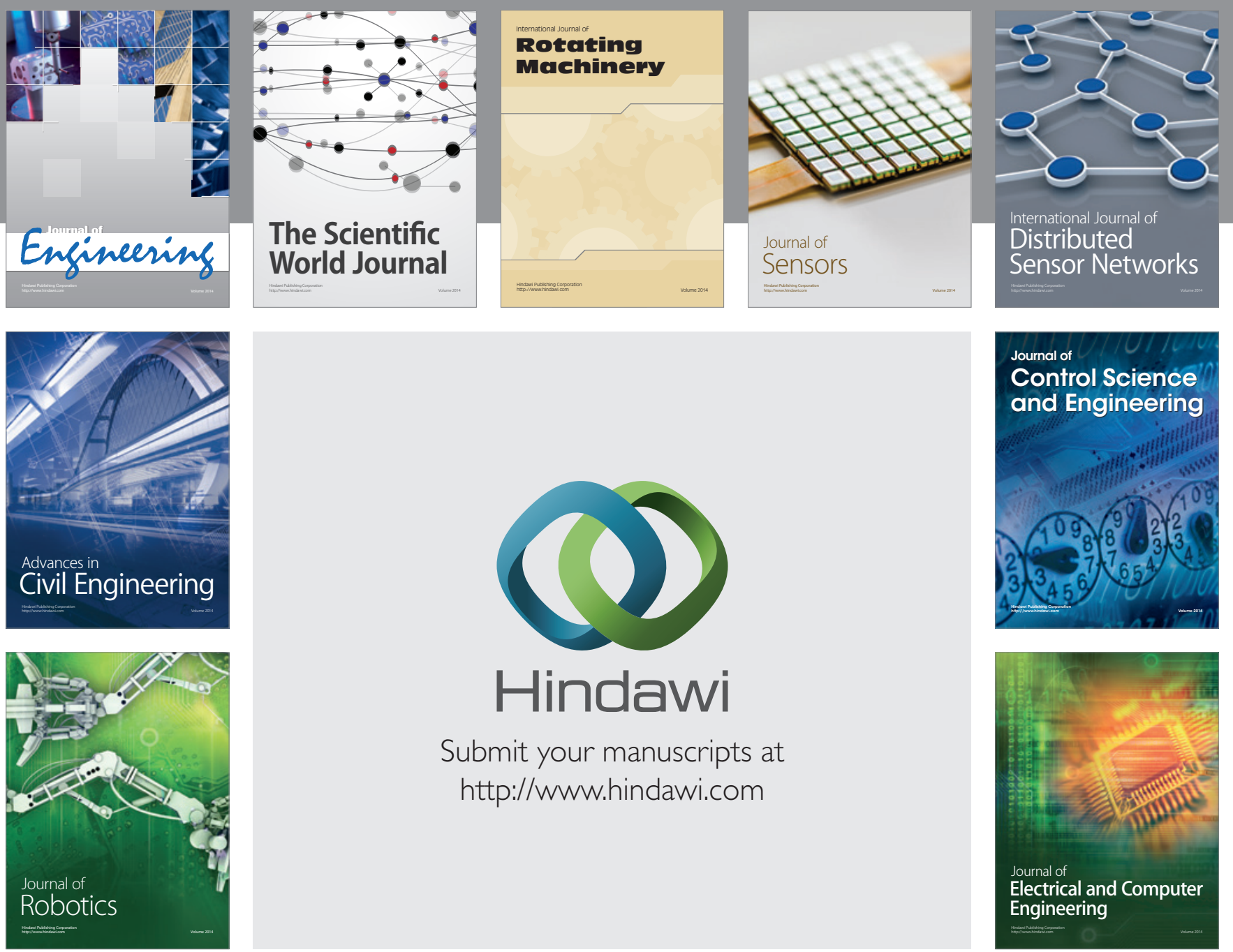

Submit your manuscripts at

http://www.hindawi.com
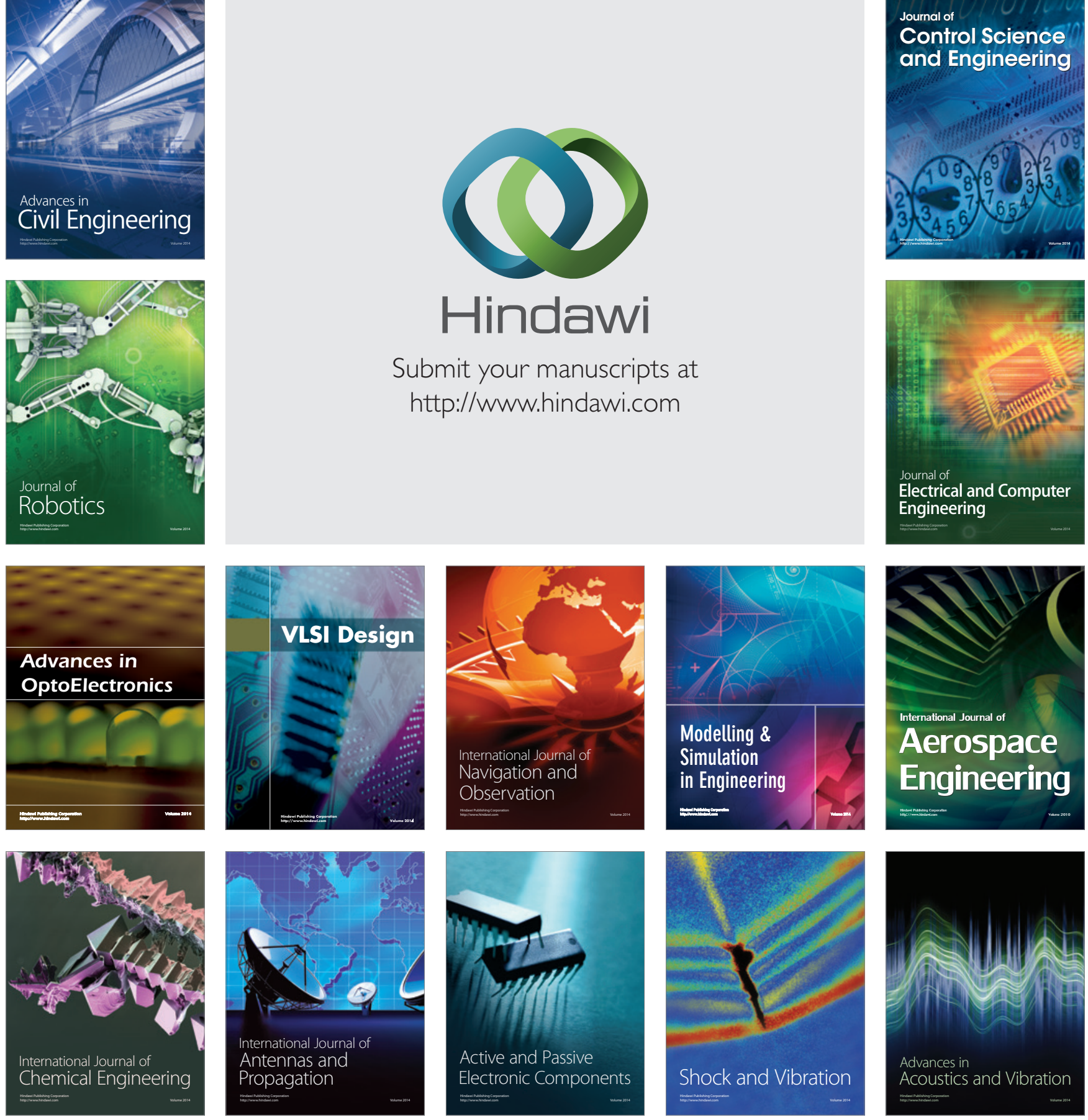